\title{
Osteonecrosis in patients with systemic lupus erythematosus develops very early after starting high dose corticosteroid treatment
}

\author{
K Oinuma, Y Harada, Y Nawata, K Takabayashi, I Abe, K Kamikawa, H Moriya
}

\begin{abstract}
Objectives-To investigate the actual time of onset of osteonecrosis (ON) after high dose corticosteroid treatment in systemic lupus erythematosus (SLE).

Methods-72 patients with active SLE, who received high dose corticosteroid for the first time, for the development of $O N$ at hips and knees were monitored by magnetic resonance imaging for at least 12 months.

Results-ON lesions were detected in $32 / 72$ patients $(44 \%)$ between one and five months (3.1 months on average) after starting high dose corticosteroid treatment. No osteonecrotic lesion was newly detected from the sixth month of treatment until the end of the follow up period. Conclusion-The findings suggested that the actual time of onset of ON in SLE is within the first month of high dose corticosteroid treatment.

(Ann Rheum Dis 2001;60:1145-1148)
\end{abstract}

It is well known that osteonecrosis (ON) frequently develops in patients with systemic lupus erythematosus (SLE) with a prevalence ranging from $10 \%$ to $52 \%{ }^{1-6}$ and that the development of ON in SLE is closely related to the high doses of corticosteroids received. Previous clinical studies have provided conflicting results about the possible association of corticosteroid treatment with the development of ON. The total treatment period, ${ }^{1}$ the highest daily dose, ${ }^{23}$ a continuous high dose, ${ }^{45}$ and the cumulative dose of corticosteroid ${ }^{6}$ have been reported as risk factors of ON in SLE. These conflicting results seem to be mainly due to the difficulties in detecting early $\mathrm{ON}$, which occurs silently and cannot be based on conventional radiographic images. Magnetic resonance imaging (MRI) has proved to be more sensitive than conventional methods, such as radiography, computed tomography, and skeletal scintigraphy, for the detection of very early, asymptomatic ON. ${ }^{78}$

We investigated the actual time of onset of ON in SLE after starting high dose corticosteroid treatment using serial MR images of both hips and both knees obtained every three months, during one year.

\section{Patients and methods}

PATIENTS

We evaluated 72 new patients with active SLE who were treated with a high dose of corticosteroid (40 mg/day or more equivalent prednisolone) at Chiba University Hospital. Thirty five of the 72 patients were also given methylprednisolone pulse treatment (MPPT). All the patients fulfilled the revised criteria for SLE established by the American Rheumatism Association. ${ }^{9}$ The study group comprised 68 women and four men with a mean age of 34.8 years (range 13-66). The disease activity of SLE was evaluated using the SLE Disease Activity Index (SLEDAI). ${ }^{10}$

We excluded patients with a history of corticosteroid use and alcoholism because it is well known that osteonecrotic lesions already exist in these patients.

\section{MRI STUDY}

MRI of both hips and both knees was carried out at $1,3,6$, and 12 months after starting corticosteroid treatment in all patients, irrespective of joint symptoms, or as close to these intervals as possible. At the end of the study follow up period, patients were divided into the $\mathrm{ON}$ group and the non-ON group based on the presence/absence of abnormal MRI findings. Abnormal MRI findings indicating osteonecrotic lesions were well demarcated, bandlike zones of decreased signal intensity on $T_{1}$ weighted, spin echo (SE) images and band-like zones of increased signal intensity on short $\tau$ inversion recovery (STIR) images. ${ }^{78} \mathrm{MRI}$ was conducted using a 0.5 Tesla superconductive unit (MRT-50; Toshiba, Japan). $\mathrm{T}_{1}$ weighted, SE images were obtained with repetition times (TR) of 300 to $400 \mathrm{~ms}$ and echo times (TE) of 18 to $40 \mathrm{~ms}$. STIR images were obtained with a TR of 1500 to $3000 \mathrm{~ms}$, inversion times $(\tau)$ of 100 to $150 \mathrm{~ms}$, and a TE of 30 to $42 \mathrm{~ms}$.

\section{STATISTICAL ANALYSIS}

To compare the clinical variables between the $\mathrm{ON}$ and non-ON groups, the Mann-Whitney $\mathrm{U}$ test or $\chi^{2}$ test was performed. Differences at $\mathrm{p}<0.05$ were regarded as significant.

\section{Results}

CHARACTERISTICS OF PATIENTS WITH OR

WITHOUT OSTEONECROTIC LESIONS

Table 1 shows the clinical profiles of patients in the ON group and non-ON group. The ON group comprised 32 patients ( 29 women, three men) with a mean age of 34.1 (range 16-66). Abnormal MRI findings were found in the hip, knee, or both. The non-ON group comprised 40 patients (39 women, one man) with a mean age of 35.1 (range 13-63). The initial mean (SD) corticosteroid dose was 58.2 (10.1) $\mathrm{mg} /$ day in the ON group and 58.6 (16.6) $\mathrm{mg} /$ day in the non-ON group. Seventeen patients in the $\mathrm{ON}$ group and 18 in the 
Table 1 Baseline characteristics of the groups with and without osteonecrosis (ON)

\begin{tabular}{lll}
\hline & ON group & Non-ON group \\
\hline Age (years) & & \\
$\quad$ Mean & 34.1 & 35.1 \\
$\quad$ Range & $16-66$ & $13-63$ \\
Sex & 29 & 39 \\
$\quad$ Female & 3 & 1 \\
$\quad$ Male & & \\
Initial corticosteroid dose $\dagger$ & $58.2(10.1)$ & $58.6(16.6)$ \\
$\quad$ (mg/day equivalent prednisolone) & $27.2(12.3)$ & $25.1(9.8)$ \\
SLEDAI $^{\star}+$ & 18 & 17 \\
MPPT $^{\star}$ & & \\
\hline
\end{tabular}

${ }^{\star}$ SLEDAI $=$ SLE Disease Activity Index MPPT $=$ methylprednisolone pulse treatment . $\dagger$ Mean (SD).

non-ON group had been treated with MPPT. The mean (SD) SLEDAI was 27.2 (12.3) in the ON group and 25.1 (9.8) in the non-ON group. There was no significant difference in age, sex, initial steroid dose, use of MPPT, or the SLEDAI between the ON and non-ON groups.

DETECTION OF OSTEONECROTIC LESIONS BY MRI In the ON group, osteonecrotic lesions were detected by MRI performed at 3.1 months of treatment on average (range 1-5) after starting high dose corticosteroid treatment (fig 1). In 14 patients (Nos 19-32) of the ON group, the MR images obtained at 92.6 (22.0) days already showed osteonecrotic lesions. On the other hand, in 18 patients (Nos 1-18) of the ON group, osteonecrotic lesions were not detected by the MR images obtained at 39.6 (19.9) days, but they appeared soon after (100.2 (27) days). No osteonecrotic lesions were found to have developed after five months. These results indicate that osteonecrotic lesions develop after 39.6 days and before 100.2 days of treatment. All osteonecrotic lesions detected by MRI showed well demarcated, band-like zones of decreased signal intensity on $\mathrm{T}_{1}$ weighted, SE images (fig 2). All patients in the ON group were clinically asymptomatic.

DISTRIBUTION OF OSTEONECROTIC JOINTS

In the ON group 92 joints had osteonecrotic lesions-44 hips in 23 patients and 48 knees in 27 patients in the ON group (fig 1). Most patients $(n=28)$ with ON had more than one joint affected-14 patients had four joints, four patients had three joints, and 10 patients had two joints. Bilaterality was common, being found in $91 \%$ of the hips and $77 \%$ of the knees.

\section{Discussion}

We believe this is the first study to investigate the actual time of onset of ON after high dose corticosteroid treatment in SLE using MRI. In this study, ON lesions, which were seen as well demarcated band-like zones, were detected by MRI at an average of 3.1 months after starting corticosteroid treatment. In a recent MRI study of patients who had received a renal transplant, ON lesions were detected at 1-4 months after starting high dose corticosteroid treatment. ${ }^{11}$ Although this study showed the presence of $\mathrm{ON}$ at the time of MRI, the actual time of onset of $\mathrm{ON}$ was not clarified.

In this paper we showed that ON lesions appeared on MRI early after starting corticosteroid treatment-namely, between 39.6 and 100.2 days of treatment. Our observations indicated that early development of ON in SLE

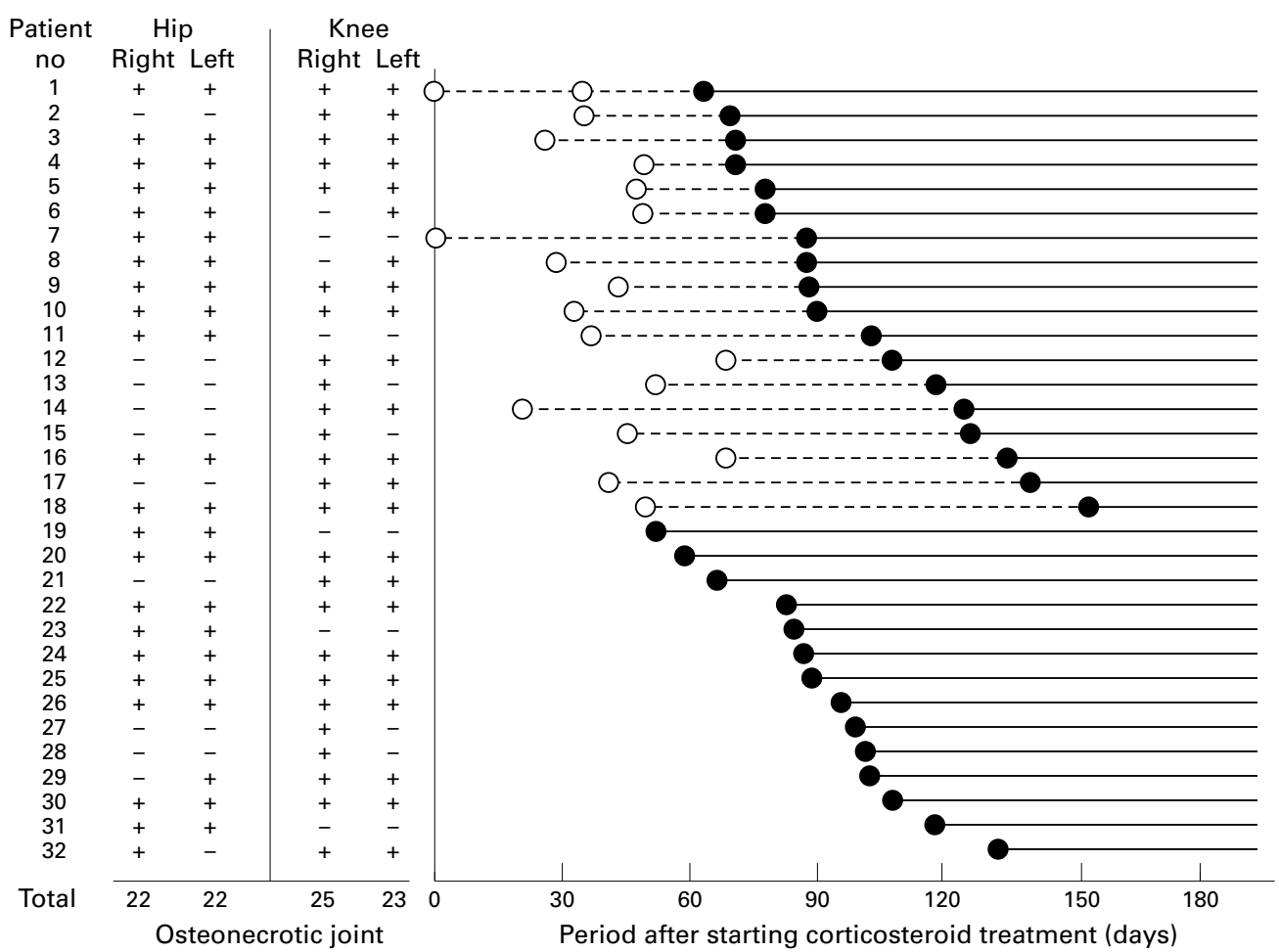

Figure 1 Distribution and detection time of osteonecrosis by magnetic resonance imaging from the start of high dose corticosteroid treatment in 32 patients. Open circle = normal finding; filled circle = abnormal finding. All these abnormalities were well demarcated, band-like zones of decreased signal intensity on $T_{1}$ weighted, spin echo images and of increased signal intensity on STIR images. 

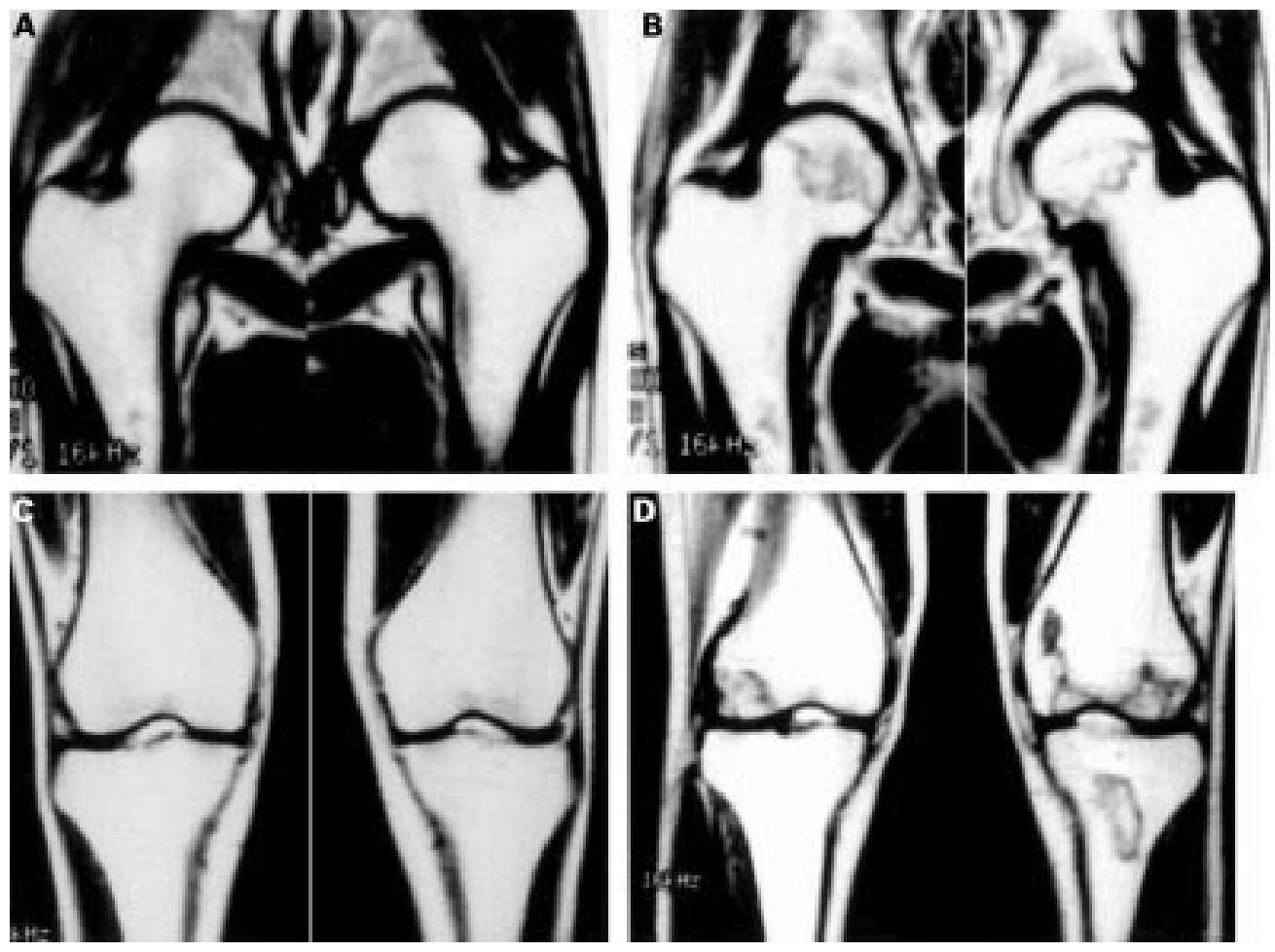

Figure 2 Patient 1. A 26 year old woman with systemic lupus erythematosus. One month after the administration of high dose corticosteroid, both the $T_{1}$ weighted, spin echo (SE) image of the hips $(A)$ and that of the knees $(C)$ showed no abnormal findings. Two months after the administration of high dose corticosteroid, both the $T_{1}$ weighted, SE images of the hips (B) and of the knees (D) showed band-like zone of decreased signal intensity. She had no symptoms in her hips or knees.

was related to an event just after high dose corticosteroid treatment, and was not related to the total treatment period, the highest daily dose, a continuous high dose, or the cumulative dose of corticosteroid. Although corticosteroid is a well known cause of ON in SLE, clinical studies attempting to demonstrate an association of corticosteroid treatment with the development of ON in SLE have yielded conflicting results. ${ }^{1-612}$ This conflict seems to be mainly caused by the employment of conventional methods, such as $x$ ray examination and bone scintigraphy, in many of the previous studies. ${ }^{78}$ Many patients with early $\mathrm{ON}$, which might have been detected by MRI, were probably missed by these conventional methods. MRI has proved to be the most useful method to detect early stage $\mathrm{ON}$ lesions that remain occult, clinically, radiologically, and scintigraphically. $^{78}$

Our study suggested that the actual onset of ON in SLE may be within the first month after starting high dose corticosteroid treatment because the band-like zones detected by MRI have been histopathologically shown to represent a reactive interface between living and dead bone, ${ }^{13}$ and thus the actual onset of ON would be one to two months before the detection of ON by MRI.

In 14 of the 32 patients with $\mathrm{ON}$ the first MRI already showed positive (fig 1, Nos 19-32). Although the assumption is that these patients already had ON before steroid treatment started, we believe that these patients should have shown normal MRI findings if they had been examined within two months after the start of corticosteroid treatment because the first MR images in 14 patients were obtained significantly later than those of the 18 remaining patients in the $\mathrm{ON}$ group (92.6 (22.0) days $v 39.6$ (19.9) days, $\mathrm{p}<0.0001)$.

We speculate that high dose corticosteroid treatment may enhance the development of $\mathrm{ON}$ as a result of microvascular ischaemia in patients with SLE who have vasculitis. It has been reported that corticosteroids decrease bone blood flow, thereby enhancing ischaemia owing to increased bone marrow pressure produced by intramedullary lipocytes hypertrophy. ${ }^{14}$ On the other hand, it was also reported that patients with SLE present haemostatic abnormalities, resulting from vascular endothelial damage, ${ }^{15}$ therefore, measurement of haemostatic variables in patients with SLE in the early period after starting high dose corticosteroid treatment may predict the subsequent development of ON. In addition, the presence of antiphospholipid antibody (aPL) has been proposed to predispose towards $\mathrm{ON}$, but its role remains controversial. ${ }^{24} 61216-18$ Recently, some large scale studies failed to show any association between aPL and ON. ${ }^{16-18}$ We also measured aPL for 45 patients in this study and found no positive relation between $\mathrm{ON}$ and the presence of aPL positivity.

In this prospective MRI study, hip and knee ON lesions occurred in $44 \%$ of patients with SLE, a percentage which was within the range reported by most other studies $(10-52 \%) .{ }^{1-6}$ All our patients with ON lesions were asymptomatic during the follow up period of 12 months, but in other reports some patients 
were symptomatic and needed surgical treatment. Segmental collapse is reported to be mainly a matter of time and depends on the size of the necrotic lesion. ${ }^{19}$ Although new osteonecrotic lesions appear after the follow up period only rarely, further follow up is recommended.

In conclusion, we found that in patients with SLE ON lesions developed very early (1-2 months) after starting high dose corticosteroid treatment. We think that MRI is useful not only in detecting early development of osteonecrotic lesions but also in examining the risk factors for their development.

1 Hurley RM, Steinberg RH, Ptariquin H, Drummond KN. Avascular necrosis of the femoral head in childhood Avascular necrosis of the femoral head in childhood
systemic lupus erythematosus. Can Med Assoc J 1974;111:781-4

2 Mont MA, Glueck CJ, Pacheco IH, Wang P, Hungerford DS. Risk factors for osteonecrosis in systemic lupus DS. Risk factors for osteonecrosis in

3 Zonana-Nacach A, Barr SG, Magder LS, Petri M. Damage in systemic lupus erythematosus and its association with corticosteroids. Arthritis Rheum 2000;43:1801-8.

4 Migliaresi S, Picillo U, Ambrosone L, Palma GD, Mallozzi $\mathrm{M}$, Tesone ER, et al. Avascular osteonecrosis in patients with SLE: relation to corticosteroid therapy and anticardiolipin antibodies. Lupus 1994;3:37-41

5 Zizic TM, Marcoux C, Hungerford DS, Dansereau JV, Stevens MB. Corticosteroid therapy associated with ischemic necrosis of bone in systemic lupus erythematosus. Am J Med 1985;36:27-35.

6 Houssiau FA, Toukap AN, Depresseux G, Maldague BE, Malghem J, Devogelaer JP, et al. Magnetic resonance imaging detected avascular osteonecrosis in systemic lupus erythematosus: lack of correlation with antiphospholipid erythematosus: lack of correlation with antiph

7 Markisz JA, Knowles RJR, Altchek DW, Schneider R, WhaMarkisz JA, Knowles RJR, Altchek DW, Schneider R, Wha-
len JP, Cahill PT. Segmental patterns of avascular necrosis of the femoral heads: early detection with MR imaging. of the femoral heads: early
Radiology 1987;162:717-20.
8 Coleman BG, Kressel HY, Dalinka MK, Scheibler ML, Cohen EK. Radiologically negative avascular necrosis: detection with MR imaging. Radiology 1988;168:525-8.

9 Tan EM, Cohen AS, Fries JF, Masi AT, McShane DJ, Rothfield NF, et al. The 1982 revised criteria for the classification of systemic lupus erythematosus. Arthritis Rheum 1982;25:1271-7.

10 Bombardier C, Gladman DD, Urowitz MB, Caron D, Chang $\mathrm{CH}$, the Committee on Prognosis Studies in SLE. Derivation of the SLEDAI: a disease activity index for lupus patients. Arthritis Rheum 1992;35:630-40

11 Kopecky KK, Braunstein EM, Brandt KD, Filo RS, Leapman SB, Capello WN, et al. Apparent avascular necrosis of the hip: appearance and spontaneous resolution of MR findings in renal allograft recipients. Radiology 1991;179:523-7.

12 Rascu A, Manger K, Kraetsch HG, Kalden JR, Manger B. Osteonecrosis in systemic lupus erythematosus, steroidinduced or a lupus-dependent manifestation? Lupus 1996; 5:323-7.

13 Lang P, Jergesen HE, Moseley ME, Block JE, Chafetz NI, Genant H. Avascular necrosis of the femoral head: high-field-strength MR imaging with histologic correlation. Radiology 1988;169:517-24

14 Wang GJ, Sweet DE, Reger SI, Thompson RC. Fat-cell changes as a mechanism of avascular necrosis of the femoral head in cortisone-treated rabbits. J Bone Joint Surg Am 1977;59:729-35.

15 Inoh $M$, Tokuda M, Kiuch H, Kurata N, Takahara J. Evaluating systemic erythematosus disease activity using molecular markers of hemostasis. Arthritis Rheum 1996;39: $287-91$

16 Sheikh JS, Retzinger GS, Hess EV. Association of osteonecrosis in systemic lupus erythematosus with abnormalities of fibrinolysis. Lupus 1998;7:42-8.

17 Mok MY, Farewell VT, Isenberg DA. Risk factors for avascular necrosis of bone in patients with systemic lupus erythematosus: is there a role for antiphospholipid antibodies? Ann Rheum Dis 2000;59:462-7.

18 Cozen L, Wallace DJ. Risk factors for avascular necrosis in systemic lupus erythematosus [letter]. J Rheumatol 1998; $25: 188$

19 Shimizu K, Moriya H, Akita T, Sakamoto M, Suguro T. Prediction of collapse with magnetic resonance imaging of avascular necrosis of the femoral head. J Bone Joint Surg Am 1994;76:215-23. 